\title{
FUNCIONES DE BIOMASA PARA BOLDO (Peumus boldus) Y ESPINO (Acacia caven) DE LA ZON:A CENTRAL DE CHILE
}

\author{
Sergio Aguirre A. (*) \\ Pedro Infante L. (*)
}

\begin{abstract}
RESUMEN
Funciones de biomasa fueron calculadas para dos importantes especies que crecen en la zona central de Chile: Boldo (Peumus boldus) y Espino (Acacia caven).

Para lograr este objetivo, se empleó una metodología de terreno y de análisis matemático que ha sido probada con éxito en otras especies nativas de la zona. Las mejores funciones obtenidas son del tipo logarítmicas para el caso del Boldo y lineales para el Espino. Considerando las buenas correlaciones y bajos errores obtenidos, estas funciones pueden utilizarse con seguridad para estimar el peso total y de los principales componentes de los árboles de Boldo y Espino.
\end{abstract}

\begin{abstract}
Regression equations were culculated for wo important species growing in the Central Zone of Chile. Proven field and analysis procedures were used. The best estimates were obtained with logarithmic functions, in the case of Boldo, and linear functions for Espino. Considering the good correlations and low calculated errors, accurate estimates from these equations can be obtained for predicting total and individual components weights of these species.
\end{abstract}

$\left.{ }^{*}\right)$ : Ingenieros Forestales. División Silvicultura. Instituto Forestal. Huérfanos 554. Piso 4. Santiago - Chile. 


\section{INTRODUCCION}

Las especies Boldo (Peumus boldus) y Espino (Acacia caven), junto con el Quillay (Quillaja saponaria) constituyen, desde el punto de vista comercial, las principales especies nativas que crecen en la zona Mediterránea de Chile Central.

El Espino se desarrolla en una formación abierta denominada Espinal, donde constituye la especie dominante, mezclada con otras especies arbóreas o arbustivas siempreverdes y sotobosque de pastos y hierbas.

La madera de esta especie es dura, pesada y muy consistente; su uso más corriente está en la producción de leña y carbón, en lo que resulta la especie más adecuada de la formación esclerófila, ya que es la que concentra el mayor porcentaje de su biomasa en las ramas gruesas (PRADO et al, 1987).

El boldo, por su parte, formaba bosques con individuos de 12 a $15 \mathrm{~m}$ de altura, pero en la actualidad no pasan de ser arbustos redondeados, originados de tocón (DONOSO, 1975). Los frutos de esta especie son comestibles, pero su uso más importante está en la explotación de sus hojas, que poseen un alcaloide denominado Boldina.

El bosque esclerófilo ha sufrido una fuerte degradación, producto de la sobreexplotación a que ha sido sometido. El Espinal, en particular. ha sido fuertemente afectado por la acción antrópica, que ha transformado gran parte de esta asociación en áreas de cultivo o praderas (DONOSO. 1982). A pesar de esto, el Espinal ocupa extensas áreas en la zona central de Chile, lo cual hace necesario disponer de una herramienta que permita estimar rendimientos y definir esquemas de manejo, cuyo objetivo sea la conservación y la obtención de una productividad sostenida de esta importante formación vegetacional. La misma necesidad se plantea en el caso del Boldo.

Las funciones de biomasa que entrega este estudio, permiten estimar, a partir de parámetros de fácil medición, la productividad física en términos de biomasa total y de hojas en el Boldo y del material leñoso, en el caso del Espino.

\section{MATERIAL Y METODO}

\section{Muestreo}

Para determinar las funciones de peso para Boldo y Espino, se empleó una muestra de 40 individuos por especie.

En el caso del Boldo. las muestras fueron obtenidas en la zona de Casablanca (V Región) y Sagrada Familia (VII Región).

Para el Espino, fueron muestradas las zonas de Rungue (Región Metropolitana) y Melipilla (Región Metropolitana).

Para asegurar una adecuada distribución de los árboles en la muestra considerada, se procedió en primer lugar a prospectar la población de cada especie. Con ello, se determinó la dispersión de la variable "diámetro mayor de copa" (DMAC), de tal forma que en la selección posterior de los árboles a muestrear para la biomasa se tomaron individuos de los diferentes tamaños presentes en la población.

De la prospección realizada, se obtuvo la distribución de árboles por clases de DMAC, que fue la base para distribuir el número de árboles a muestrear en cada especie.

En cada caso, 20 árboles fueron asignados en forma equitativa en las clases y los 20 restantes fueron distribuidos en forma aleatoria, pero con probabilidad proporcional a altura de 
máximo follaje, multiplicado por diámetro mayor de copa (HMF x DMAC), lo que tiene por objeto dar una mayor probabilidad de asignación a aquellas clases de mayor desarrollo. El número de árboles-muestra según clase de DMAC se presenta en los Tablas 1 y 2.

TABLA 1

DISTRIBUCION DE LOS ARBOLES-MUESTRAS DE BOLDO

\begin{tabular}{|c|c|c|}
\hline Clase & Diámetro mayor de copa & Arboles-muestra \\
\hline & $1.6-2.5$ & 4 \\
2 & $2.6-3.5$ & 8 \\
3 & $3.6-4.5$ & 8 \\
4 & $4.6-5.5$ & 12 \\
5 & $5.6-6.5$ & 8 \\
\hline TOTAL & & 40 \\
\hline
\end{tabular}

TABLA 2

DISTRIBUCION DE LOS ARBOLES-MUESTRAS DE ESPINO

\begin{tabular}{|c|c|c|}
\hline Clase & Diámetro mayor de copa & Arboles-muestra \\
\hline & & \\
2 & $1.7-2.4$ & 6 \\
3 & $2.5-3.2$ & 6 \\
4 & $3.3-4.0$ & 6 \\
5 & $4.1-4.8$ & 14 \\
\hline TOTAL & $4.9-5.7$ & 8 \\
\hline
\end{tabular}

Obtención de peso verde y peso seco.

Luego de seleccionados los individuos a muestrear, se procedió a medir las variables independientes del árbol, que parecían ser las más relacionadas con el peso. Para Boldo se midieron:

- Diámetro mayor de copa

- Diámetro menor de copa

- Altura total

- Altura de máximo follaje

- Número de retoños mayores de $1 \mathrm{~cm}$

- Diámetro en la base (cepa)

$\begin{array}{ll}(\mathrm{DMAC}) & (\mathrm{m}) \\ (\mathrm{DMEC}) & (\mathrm{m}) \\ (\mathrm{HT}) & (\mathrm{m}) \\ (\mathrm{HMF}) & (\mathrm{m}) \\ (\mathrm{NR}) & \\ (\mathrm{DB}) & (\mathrm{cm})\end{array}$

En el caso de Espino, se midieron las mismas variables anteriores, agregándose además la medición del diámetro en la base de los retoños o fustes (DR) $(\mathrm{cm})$.

Una vez realizadas las mediciones indicadas, los árboles-muestra fueron cortados e inmediatamente pesados. 
En el caso de Boldo, la biomasa fue dividida en dos componentes: hojas y ramas, que fueron consideradas en forma separada. Sin embargo, ante la dificultad de realizar en forma directa el pesaje de total de las hojas, en cada árbol de Boldo se tomó una submuestra de 3 vástagos representativos, donde se pesaron en forma separada las hojas y las ramas. Con ello, se obtuvo una relación peso hojas/peso ramas, que fue aplicada luego al peso total, obteniéndose así el peso de las hojas de cada árbol.

En Espino, el árbol fue dividido también en dos componentes: ramas y ramillas. Las ramas constituyen el material leñoso con un diámetro mínimo de $3 \mathrm{cms}$, y las ramillas, aquel material de menos de $3 \mathrm{~cm}$ de diámetro, incluyendo hojas y frutos.

Finalmente, para el cálculo del peso seco de cada árbol, se tomaron submuestras de los componentes de cada árbol y se enviaron a laboratorio para el secado hasta obtener peso constante. Así se obtuvo relación peso seco/peso verde.

\section{Análisis}

Para expresar las relaciones entre peso y las dimensiones de los árboles muestreados, se probaron varios modelos, empleando las variables independientes tal como fueron medidas, combinadas o transformadas $(\ln )$. Se probaron los siguientes modelos:

i) Alométricos, con las siguientes variaciones:

$$
\begin{aligned}
& Y=b_{0} X^{b 1} e \\
& Y=b_{0}\left(X_{1}{ }^{2} X_{2}\right)^{b 1} e \\
& Y=b_{0} X_{1}{ }^{b l_{1}} X_{2}{ }^{b 2} e
\end{aligned}
$$

Todos estos modelos fueron ajustados mediante transformación logarítmica, asumiendo que el error (e) es multiplicativo (BASKERVILLE, 1972) y por lo tanto corregidos de acuerdo al procedimiento dado por WIANT y HARNER (1979) para compensar el sesgo producido por la transformación.

ii) Lineales de forma:

$Y=b_{0}+b_{1} X_{1}+b_{2} X_{2}+\ldots \ldots \ldots \ldots+b_{n} X_{n}$

La selección de las variables a ser incluidas en cada función, se hizo empleando el método de regresión paso a paso (step-wise).

Para seleccionar las mejores funciones, se compararon los valores del Coeficiente de Determinación ( $\left.\mathrm{R}^{2}\right)$, los Errores Caudráticos Medios (ECM), y la proporción de observaciones con errores menores al 10,15 y $20 \%$. 


\section{RESULTADOS Y DISCUSION}

Las funciones seleccionadas para estimar peso seco total y de los componentes de Boldo y Espino, se muestran en las Tablas siguientes:

TABLA 3

FUNCIONES DE BIOMASA PARA ESTIMAR PESO SECO TOTAL Y DE COMPONENTES DEL BOLDO (Peso en Kg)

\begin{tabular}{|c|c|c|c|}
\hline Componente & Función de Peso & $\mathbf{R}^{2}$ & $\underset{(\%)}{\mathbf{E C M}}$ \\
\hline $\begin{array}{l}\text { HOJAS } \\
\text { RAMAS } \\
\text { TOTAL }\end{array}$ & $\begin{array}{l}\ln (\mathrm{PHOJ})=-(1.2379+1.2412 \ln (\mathrm{DMEC})+0.2627 \ln (\mathrm{NR} \times \mathrm{HT}) \\
\ln (\mathrm{PRAM})=-0.1403+1.0276 \ln (\mathrm{DMAC} \times \mathrm{DMEC} \times \mathrm{HT}) \\
\ln (\mathrm{PTOT})=-0.0837+0.7657 \ln (\mathrm{DMAC} \times \mathrm{DMEC} \times \mathrm{HT})+0.1638 \ln \\
\left(\mathrm{HT}^{2} \times \mathrm{DMEC} \times \mathrm{NR}\right)\end{array}$ & $\begin{array}{l}0.78 \\
0.92 \\
0.92\end{array}$ & $\begin{array}{l}32.7 \\
29.3 \\
25.7\end{array}$ \\
\hline $\begin{array}{ll}\ln & = \\
\text { PHOJ }= \\
\text { PRAM }= \\
\text { PTOT }= \\
\mathrm{R}^{2}= \\
\text { ECM }=\end{array}$ & $\begin{array}{lrl}\text { Logaritmo natural } & \text { DMEC } & =\text { Diámetro menor copa }(\mathrm{m}) \\
\text { Peso seco hojas }(\mathrm{kg}) & \mathrm{NR} & =\text { Número de retonos mayores de } \\
\text { Peso seco ramas }(\mathrm{kg}) & \mathrm{HT} & =\text { Altura total }(\mathrm{m}) \\
\text { Peso seco total }(\mathrm{kg}) & \text { DMAC } & =\text { Diámetro mayor de copa }(\mathrm{m}) \\
& \text { Coeficiente de determinación } & \end{array}$ & $\mathrm{cm}$ & \\
\hline
\end{tabular}

TABLA 4

FUNCIONES DE BIOMASA PARA ESTIMAR PESO SECO TOTAL Y DE COMPONENTES DEL ESPINO (Peso en Kg)

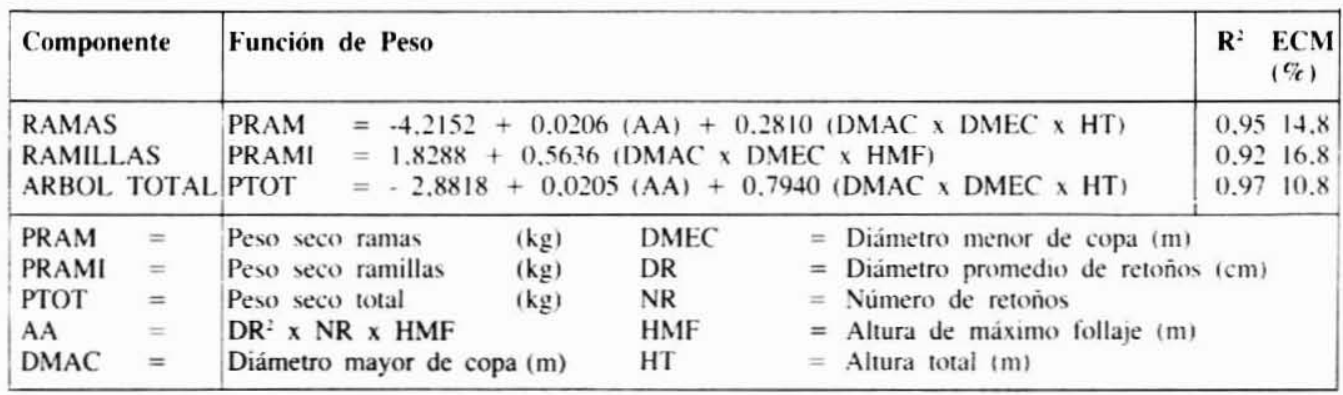

En el caso del Boldo, las mejores funciones para la estimación del peso seco, son del tipo logarítmicas y presentan correlaciones y errores aceptables, considerando la gran variabilidad que presenta la especie. El principal componente del árbol, las hojas, es estimado mediante una función que considera variables de fácil medición, como son: diámetro menor de copa (DMEC), número de retonoos mayores de $1 \mathrm{~cm}$ de diámetro (NR) y altura total (HT).

Las funciones calculadas para la estimación del peso seco de Espino, pueden considerarse como buenos estimadores. En el caso de las ramas, que es el principal componente de esta especie, la mejor función considera la medición de seis variables. A pesar que estas variables 
son en general de fácil medición, se obtuvo una función alternativa, que aunque tiene una correlación un poco más baja, emplea solamente tres variables (diámetro promedio de retoños (DR), número de retoños (NR) y altura de máximo follaje (HMF), lo que facilita un inventario de biomasa. Esta función de peso seco de ramas es la siguiente:

$$
\begin{array}{r}
\text { PRAM }=0.0519+0.0292\left(\mathrm{DR}^{2} \times \mathrm{NR} \times \mathrm{HMF}\right) \\
\mathrm{R}^{2}=0.93 \quad ; \quad \mathrm{ECM}=19.2 \%
\end{array}
$$

Finalmente, respecto a la distribución de la biomasa en el árbol, en el caso del boldo, la mayor parte de ella ( $84 \%$ del peso seco), se encuentra en las ramas y ramillas, es decir, en la parte leñosa del árbol y sólo un $16 \%$ del peso corresponde a las hojas, que constituye el componente comercial.

En Espino, las ramas con diámetro mayor de $3 \mathrm{~cm}$ concentran el $56 \%$ del peso seco del árbol. lo que corrobora la aptitud de la especie en la producción de leña y carbón.

\section{CONCLUSIONES}

A pesar de la variabilidad que presentan las especies Boldo y Espino, en cuanto a forma, edad y estado de alteración, las funciones de biomasa calculadas se estiman aceptables y pueden ser aplicadas con seguridad, dentro de los rangos de las variables consideradas. (Tablas 1 y 2 ).

Dada la heterogeneidad que presentan las especies estudiadas debido a su amplia distribución. la confiablidad de las estimaciones puede disminuir, si las funciones se aplican en áreas sin características muy diferentes a las zonas muestreadas.

Se estima que las funciones que entrega este trabajo, permiten hacer estimaciones confiables de la biomasa de arboles de monte bajo, en la zona central del país (V-VII Regiones).

\section{AGRADECIMIENTOS}

Este trabajo fue desarrollado con datos obtenidos de proyectos contratados al Instituto Forestal por el Centro Internacional de Investigaciones para el Desarrollo (CIID) Canadá, y el Proyecto CONAF/FAD/PNUD CHIB -017 .

\section{REFERENCIAS BIBLIOGRAFICAS}

I. BASKERVILLE. G.L. 1972. Use of logarithmic regression in the estimation of plant biomasa. Can. J. For. Res 2: $49-53$.

2. DONOSO Z., CLAUDIO. 1975. Dendrología árboles y arbustos chilenos. Universidad de Chile, Fac. Ciencias Forestales, Manual $\mathrm{N}^{\circ} 2,141$ pág.

3. DONOSO Z. CLAUDIO. 1982. Reseña ecológica de los bosques mediterráneos de Chile. Universidad Austral de Chile. Fac. Cs, forestales. Bosque 4 (2).

4. PRADO, J.A., INFANTE, P., ARRIAGADA M., y AGUIRRE S. 1987. Funciones de biomasa para seis especies arbustivas de la Zona Arida Chilera Ciencia e Investigación Forestal 1(2): 11 - 20.

5. WIANT, H.V. and E.J HARNER. 1979. Percent bias and standard error in logarithmic regression. For. Sci. 25(1): $167 \cdot 168$. 